\title{
"To be or not to be" for PET in rheumatology. A marriage of love or of convenience?
}

\author{
Leszek Królicki \\ Department of Nuclear Medicine, Medical University of Warsaw, Poland
}

The mechanisms responsible for the development and course of rheumatic diseases are not yet fully explained. Molecular imaging is one of the tools used to investigate them, with nuclear medicine being its prominent part.

Unlike computed tomography (CT), ultrasonography (USG), or magnetic resonance imaging (MRI), which reveal only structural disorders, radioisotope imaging enables us to present the functional changes. Its methods are characterised by high sensitivity - radioisotope examination can reveal changes in substance concentration as small as $10^{-11} \mathrm{mmol}$, while MRI, respectively, $10^{-3}-10^{-4}$ mol. This means that radioisotope methods can be used to effectively trace the changes in concentrations of chosen chemical compounds while they take place in the course of the disease. Thus the assessment of functional changes becomes possible, which should be essential for the choice of proper therapy.

Nuclear medicine methods meet numerous vital requirements determined by the standards for modern medical science, such as:

- They enable early diagnosis of the changes caused by the disease and allow us to establish their nature. The diagnosis should precede the emergence of irreversible structural damage.

- They can be used for the precise establishment of the state of progress of pathologic neoplastic changes.

- The employed imaging methods should enable us to predict the course of the disease and use a personalised treatment to counter the illness.

- As the costs of modern therapies increase, it should be possible to use the diagnostic methods to evaluate the effectiveness of ongoing therapy.

- Modern medicine requires diagnostic methods that can definitely determine the disease regression and reveal its recurrence as early as possible.

Nuclear medicine has proven to be effective in solving all of the above listed clinical issues; moreover, it meets pharmacoeconomic cost-effectiveness criteria.

Fluorodeoxyglucose $\left({ }^{18} \mathrm{FDG}\right)$ is a radiopharmaceutical most commonly used in PET examinations. It is an analogue of glucose. The areas of the increased marker accumulation are evidence of intensified metabolism associated with raised glucose demand. This can be a result of neoplasmic or of inflammatory processes. The increased ${ }^{18} \mathrm{FDG}$ accumulation is an effect of the increased activity of Glut1 and Glut 3 transport mechanisms, as well as with the elevated activity of hexokinase. These phenomena are typical for cells undergoing rapid proliferation, e.g. the macrophages, neutrophils, and new granulation tissue.

${ }^{18}$ FDG-PET examination allows for the assessment of the inflammation progress. Matsui et al. [1] demonstrated in their in vitro research that the highest accumulation of ${ }^{18} \mathrm{FDG}$ occurs in the course of the pannus creation and destruction of the bone structure by inflammation. This process is the effect of fibroblast proliferation. The role of the macrophages must also be noticed: the accumulation of ${ }^{18}$ FDG by unactivated macrophages is minimal, but after their activation by hypoxia or tumour necrosis factor $\alpha$ (TNF- $\alpha$ ), their demand for glucose increases significantly. Beckers et al. [2] showed that the sensitivity of the described method in rheumatoid arthritis is $90 \%$. A number of publications indicate that ${ }^{18} \mathrm{FDG}$-PET is able to reveal pathologic changes in their subclinical stage or during clinical remission. This may play a vital role in the therapeutic proceedings [3]. PET can also be used to evaluate the stage of progress of pathologic changes in spondyloarthritis, polymyalgia rheumatica, Still's disease, polychondritis, IgG4-related disease, polymyositis, and dermatomyositis [4].

A number of publications point to the fact that ${ }^{18} \mathrm{FDG}$ is a very efficient marker for therapy effectiveness assessment. Elisng et al. [5] demonstrated that the decrease in ${ }^{18} \mathrm{FDG}$ uptake, subsequent to the start of the anti-TNF drug therapy, is an important prognostic factor for the effectiveness of treatment.

It is important to stress the additional aspect of PET - the diagnosis of neoplastic illnesses coexisting with lupus erythematosus, systemic sclerosis, dermatomyositis/ polymyositis, or Sjögren's disease. Epidemiological data 
indicate that in the group of patients with these diseases, neoplastic illnesses occur significantly more often than in the general population; the increased frequency of such patients being affected by lymphoma, oesophageal, and pancreatic cancer has been demonstrated [6-8].

The treatment of autoimmunological disorders includes the use of immunosuppressive drugs, which limit the organism's immune response, and thus create an increased risk of infection, among others - tuberculosis relapse [9]. ${ }^{18} \mathrm{FDG}-\mathrm{PET}$ has proven itself to be the most sensitive examination in the diagnosis of infections. ${ }^{18} \mathrm{FDG}$-PET presents the foci of pathologic accumulation of the marker in $90 \%$ of patients suffering from autoimmunological disorders; the sensitivity and specificity of this method in the diagnosis of neoplasm is, respectively, $100 \%$ and $67 \%$ [10].

Another group of patients, for whom the ${ }^{18}$ FDG-PET examination has also proven to be very useful, is patients with symptoms of fever of unknown origin. In this group, in which other imaging techniques are undiagnostic, ${ }^{18} \mathrm{FDG}$-PET can localise the area of inflammation/neoplasm in $47 \%$ of patients. The positive predictive value of this examination has been established at $78 \%$, and negative - at 88\% [11].

An important indication for performing the described test is the suspicion of large vascular vessel (LVV) vasculitis (Fig. 1). This term encompasses a number of illnesses, with Takayasu's disease and giant cell arthritis (GC A) being the most frequent of them. The sensitivity and specificity of ${ }^{18}$ FDG-PET in the diagnosis of LVV vasculitis reaches $90 \%$. In a meta-analysis presented by Balinka et al. the authors stress that the sensitivity of ${ }^{18} \mathrm{FDG}$-PET is higher than that of other diagnostic methods [12].

Choline is an another marker of the proliferative processes taking place in the course of the disease. It

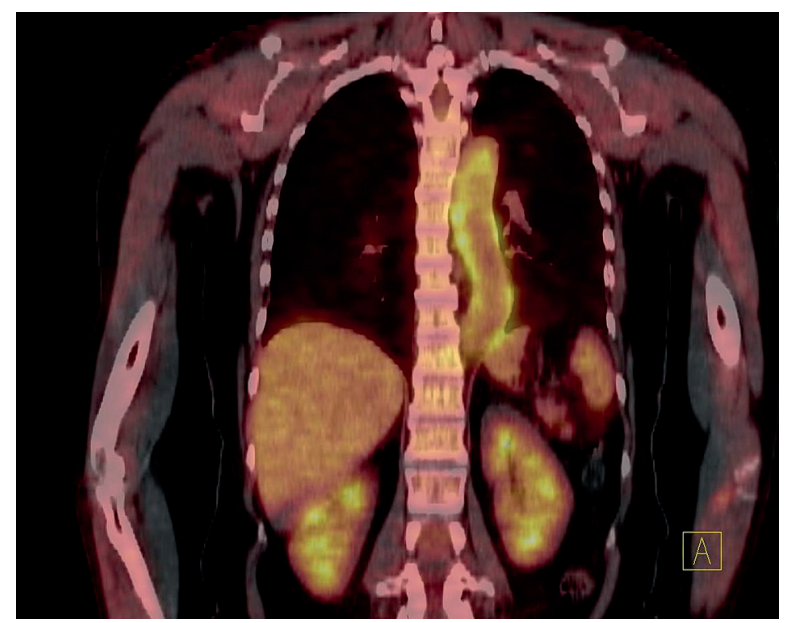

Fig. 1. Inflammation of the aortic wall in 18FDG$\mathrm{PET} / \mathrm{CT}$ examination. is taken up by the rapidly dividing cells and then phosphorylated, by choline kinase, to phosphorylcholine. This compound, in turn, is necessary for the phospholipid synthesis, phosphatidylcholine - a building material of the cellular membranes - in particular. Its increased accumulation depends of mechanisms of active transport through the cellular membrane and of passive diffusion taking place in the hyperaemic disease foci. It has been demonstrated that the said marker accumulates in the initial phase of the inflammation. Its accumulation correlates with the level of the enhancement in MRI post-gadovist application and with the accumulation of ${ }^{18} \mathrm{FDG}$ [13]. The accumulation of ${ }^{18} \mathrm{FDG}$ or ${ }^{18} \mathrm{~F}$-choliny in inflammatory tissue is non-specific. These markers accumulate also in the neoplasmic foci. Due to this fact, research into the application of markers specific for the inflammation taking place in the rheumatic diseases is being performed.

One of the new substances proposed for the use in PET is (R)-[11C]PK11195 isoquinolone carboxamide [14]. This radiopharmaceutical binds to monocytes and macrophages. It is an antagonist of peripheral benzodiazepine receptor (PBR). Currently this peptide bears the name translocator protein (TSPO). It can be particularly active at the external surface of the mitochondrial membrane of the activated macrophages and multinuclear cells, as well as in the nervous and lymphatic tissues. It is responsible for the steroidogenesis, apoptosis, cellular proliferation, and immunological processes. Initially it was demonstrated that this radiopharmaceutical is an effective neuroinfection marker (the peripheral benzodiazepine receptors are present on the activated microglia cells). Yet, this marker can also be used for the assessment of the stage of rheumatologic arthritis progression. The level of the marker accumulation in PET examination correlates with the intensity of synovitis assessed in the histopathological examination (the level of the macrophage infiltration).

The clinical trials of radiopharmaceutical marking:

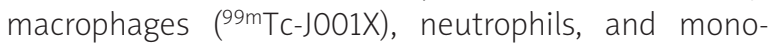

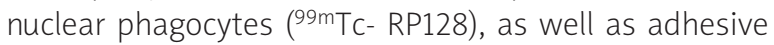

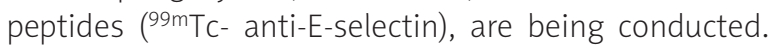
Somatostatin analogues and monoclonal antibodies are

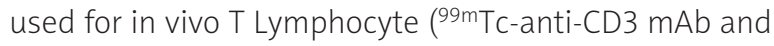
99mTc-anti-CD4) and B Lymphocyte ( ${ }^{99 m T c-a n t i-C D 20)}$ marking. Numerous observations suggest the possibility of apoptosis ( ${ }^{99 \mathrm{~m} T c-A n n e x i n ~ V)}$ ) and cytokine activity ( ${ }^{99 m}$ Tc-anti-TNF- $\alpha$ ) assessment.

The above described array of radiopharmaceuticals shows many possibilities of radioisotope technique application in the diagnosis of rheumatic diseases [15].

The title of my editorial ends with a question mark but should it really be placed there? I am convinced that 
as the "marriage" of rheumatology to MRI, CT, and USG is one of a conventional nature, its relationship with the radioisotope examination is based on a strong mutual need for each other. Complex processes, such as the ones taking place in rheumatological diseases, require comprehensive information, which can be acquired with the methods that only nuclear medicine can provide.

The author declares no conflict of interest.

\section{References}

1. Matsui T, Nakata N, Nagai S, et al. Inflammatory cytokines and hypoxia contribute to $18 \mathrm{~F}-\mathrm{FDG}$ uptake by cells involved in pannus formation in rheumatoid arthritis. J Nucl Med 2009; 50: 920-926.

2. Beckers C, Ribbens C, André B, et al. Assessment of disease activity in rheumatoid arthritis with (18)F-FDG PET. J Nucl Med 2004; 45: 956-964.

3. Brown AK, Conaghan PG, Karim Z, et al. An explanation for the apparent dissociation between clinical remission and continued structural deterioration in rheumatoid arthritis. Arthritis Rheum 2008; 58: 2958-2967.

4. Yamashita H, Kubota K, Mimori A. Clinical value of whole-body PET/CT in patients with active rheumatic diseases 2014. Arthritis Res Ther 2014; 16: 423.

5. Elzinga EH, van der Laken CJ, Comans EF, et al. 18F-FDG PET as a tool to predict the clinical outcome of infliximab treatment of rheumatoid arthritis: an explorative study. J Nucl Med 2011; 52: 77-80.
6. Kiss E, Kovacs L, Szodoray P. Malignancies in systemic lupus erythematosus. Autoimmun Rev 2010; 9: 195-199.

7. O'Byrne KJ, Dalgleish AG. Chronic immune activation and inflammation as the cause of malignancy. Br J Cancer 2001; 85 : 473-483.

8. Sela O, Shoenfeld Y. Cancer in autoimmune diseases. Semin Arthritis Rheum 1988; 18: 77-87.

9. Carter PH, Zhao Q. Clinically validated approaches to the treatment of autoimmune diseases. Expert Opin Investig Drugs 2010; 19: 195-213.

10. Oh JR, Song HC, Kang SR, et al. The clinical usefulness of 18F-FDG PET/CT in patients with systemic autoimmune disease. Nucl Med Mol Imaging 2011; 45: 177-184.

11. Ergül N, Çermik TF. FDG-PET or PET/CT in fever of unknown origin: the diagnostic role of underlying primary disease. Int J Mol Imaging 2011; 2011: 318051.

12. Balink H, Bennink RJ, van Eck-Smit BL, Verberne HJ. The role of 18F-FDG PET/CT in large-vessel vasculitis: appropriateness of current classification criteria? Biomed Res Int 2014; 2014: 687608.

13. Roivainen A, Parkkola R, Yli-Kerttula T, et al. Use of positron emission tomography with methyl-11C-choline and 2-18F-fluoro-2-deoxy-D-glucose in comparison with magnetic resonance imaging for the assessment of inflammatory proliferation of synovium. Arthritis Rheum 2003; 48: 3077-3084.

14. van der Laken CJ, Elzinga EH, Kropholler MA, et al. Noninvasive imaging of macrophages in rheumatoid synovitis using 11C-(R)PK11195 and positron emission tomography. Arthritis Rheum 2008; 58: 3350-3355.

15. Zeman MN, Scott PJ. Current imaging strategies in rheumatoid arthritis. Am J Nucl Med Mol Imaging 2012; 2: 174-220. 